\title{
Spectral luminous efficiency functions for a ten-degree field in the mesopic range
}

\author{
Ken SAGAWA* and Keishiro TAKEICHI*
}

\begin{abstract}
Mesopic photometry is one of the current problems of the CIE TC-1.4. The Iuminous efficiency function for mesopic vision has not been established yet, whereas the functions $V(\lambda)$ for photopic vision and $V^{\prime}(\lambda)$ for scotopic vision have been already standardized. In the present study, we measured the spectral luminous efficiency functions in the mesopic range extensively to offer basic data for making photometry more visually meaningful. A direct brightness matching method of $10^{\circ}$ field was employed. White light was used as a reference stimulus, and its retinal illuminance was varied from 0.01 to 100 photopic trolands in $0.5 \mathrm{log}$ unit steps to cover the mesopic range. Spectral luminous efficiency function from 400 to $700 \mathrm{~nm}$ in $10 \mathrm{~nm}$ steps was measured for each retinal illuminance level. Data of seven subjects (19-23 yr old) were collected.

It was shown that the function obtained changed gradually from the photopic function to the scotopic one as retinal illuminance decreased. The average and individual variation of seven subjects' data were presented as basic data of luminous efficiency for mesopic vision. The change of the function due to the different contributions of photopic and scotopic functions was discussed according to the Ikeda-Shimozono's formula.
\end{abstract}

\section{Introduction}

The measurement of light in the mesopic range has become much important problem in our everyday-life as we have much time to be in the mesopic visual environment with the development of illuminating engineering. However, the photometry system for mesopic vision has not been established by the CIE, while those for photopic and scotopic vision have been already standardized. One reason for the difficulty of establishing mesopic photometry is the complicated change of luminous efficiency function with luminance level because of the different contributions of rod and cone systems to brightness perception, and the basic data to specify the change of the function is still lacking in the literature. Although some sets of data have been already reported ${ }^{1) \sim 3}$, those data do not seem to be sufficient to meet the criterion for establishing a standard data in view points of number of subjects, luminance range, and spectral range. To establish standard luminous efficiency data in mesopic range is indispensable for developing mesopic photometry system.

On the other hand, some empirical formulae or vision models to express the mesopic function by using photopic and scotopic functions, for example existing $V(\lambda)$ and $V^{\prime}(\lambda)$, have been postulated. To evaluate those formulae more precisely, extensive data for luminous efficiency functions for mesopic vision are much needed.

\footnotetext{
* Human Factors Engineering Division, Industrial Products Research Institute, 1-1-4, Yatabe-machi Higashi, Tsukuba, Ibaraki 305, Japan
}

The purpose of this study is to provide basic data of luminous efficiency functions for mesopic vision, which will be used as the fundamental data for photometry system. These basic data are based on the direct brightness matching of $10^{\circ}$ field using a number of subjects, wide luminance range covering photopic, mesopic, and scotopic vision, and wide spectral range. The average efficiency data are given in a figure and a table as a standard at present and the individual variations of the data are presented in a figure.

\section{Method}

\section{Apparatus}

Figure 1 shows a schematic diagram of the apparatus employed in the experiment. Two-channel Maxwellian view optical system was used with a $500 \mathrm{~W}$ xenon lamp as a light source. One channel provided a white reference stimulus in an upper half of a $10^{\circ}$ bipartite field which was viewed centrally. The retinal illuminance of the stimulus was varied from 100 to 0.01 photopic trolands in $0.5 \mathrm{log}$ unit steps by neutral filters (ND1) and an optical wedge (W1). The spectral energy distribution for the $100 \mathrm{td}$ reference stimulus is shown as the top curve in Fig. 2. The energy distributions for the other retinal illuminance levels were slightly different from that of the $100 \mathrm{td}$ because of non-neutral transmittances of the neutral filters and the optical wedge. The energies for the shortwave region were relatively lower than those for the long-wave region as retinal illuminance decreased. The energy distributions for the $1 \mathrm{td}$ and the $0.01 \mathrm{td}$ levels are also shown in Fig. 2.

A test stimulus was produced by another channel 


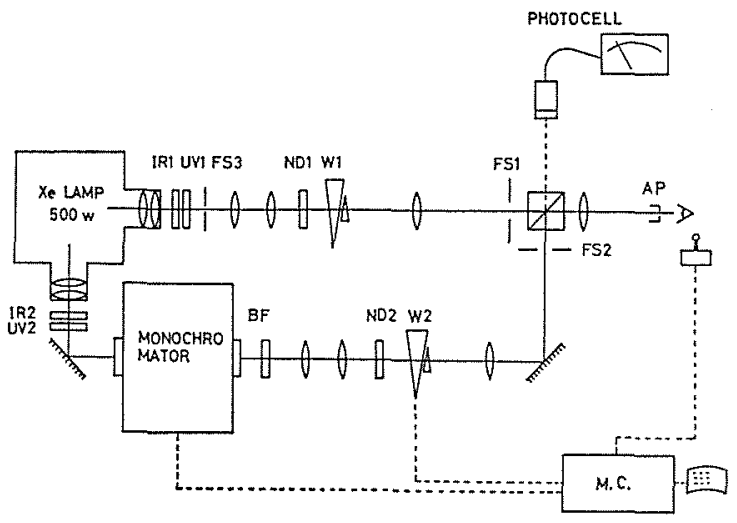

Fig. 1 Schematic diagram of the apparatus. ND1, ND2: neutral density filter; W1, W2: optical wedge; IR1, IR2: infrared-cut filter; UV1, UV2: ultraviolet-cut filter; $B F$ : blocking filter; FS1, FS2, FSs: field stop; AP: artificial pupil; M.C.: microcomputer.

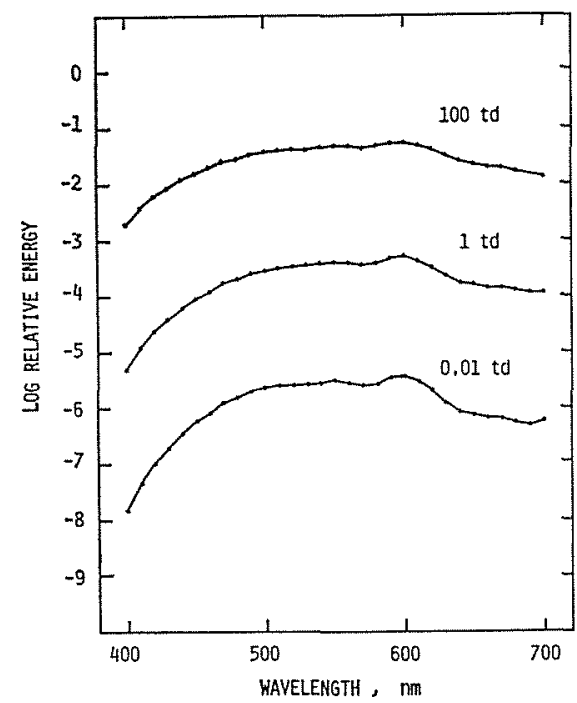

Fig. 2 Relative spectral energy distributions of a white reference stimulus at the 100 , 1 , and $0.01 \mathrm{td}$ levels.

which had a single grating monochromator to produce monochromatic lights. Monochromatic lights from 400 to $700 \mathrm{~nm}$ in $10 \mathrm{~nm}$ steps were used as test stimuli for all the retinal illuminance levels examined. Half band-widths of the monochromatic lights were $6 \mathrm{~nm}$. A wratten $47 \mathrm{~A}$ filter for $400 \sim$ $440 \mathrm{~nm}$ and a $670 \mathrm{~nm}$ sharpcut filter for $670 \sim 700 \mathrm{~nm}$ were used as blocking filters (BF) to avoid undesirable stray lights in the monochromator. Radiant energy of test light was controlled by neutral filters (ND2) and an optical wedge (W2). Monochromator and the optical wedge in test channel were driven under the control of a microcomputer (M.C.) and the wavelength of the monochromator and the position of the wedge were automatically recorded. Radiant energies of both the test and the reference stimuli were monitored throughout the experiment by a silicon photodiode fixed in the optical system. An artificial pupil of $3 \mathrm{~mm}$-diameter (AP) was used. Subject's head was fixed by a dental impression bite-bar.

\section{Calibration}

The relative energies of test stimuli were measured with a silicon photodiode and a photomultiplier which had been calibrated against the standard silicon photodiode of Electrotechnical Laboratory in Japan. Spectral transmittances of neutral filters and spectral attenuation rates of the wedges were calibrated in the optical system by using the silicon photodiode and the photomultiplier. Wavelength calibration of the monochromator was performed by verifying the maximum transmittances of 435.8 and $546.1 \mathrm{~nm}$ beams of $\mathrm{Hg}$ lamp.

Retinal illuminance of the white reference stimulus was measured by an illuminance meter placed at the artificial pupil. This measurement was carried out with the wedge set at the position of the highest transmittance and without neutral filters, so that the maximum retinal illuminance of the optical system was obtained. Retinal illuminances of the other low levels used in the experiment were estimated by spectral optical densities of the neutral filters and the wedge which were previously calibrated.

\section{Procedure}

It is well known that the luminous efficiency function critically depends on the experimental condition such as visual field size and method of measurement. In mesopic vision, brightness perception of a relatively large field seems to be important since we do not see the details of objects because of low visual acuity in this condition. Therefore, in the present experiment, a direct brightness matching method of $10^{\circ}$ field was employed.

Spectral luminous efficiency function for each retinal illuminance level was measured by asking the subjects to adjust the energy of the test stimulus until it looked equally bright to the reference stimulus. The test and reference stimuli were present steadily throughout the experiment. Nine retinal illuminances of the reference stimulus i.e. $100,32,10,3.2,1,0.32,0.1,0.032$, and $0.01 \mathrm{td}$, were used. The retinal illuminance in each experimental session was varied in two series, the decreasing series from 100 to $0.01 \mathrm{td}$ and the increasing series from 0.01 to $100 \mathrm{td}$. A total of 18 sessions were carried out for each subject. In the decreasing series, test stimuli were presented in wavelength order from 400 to $700 \mathrm{~nm}$ in $10 \mathrm{~nm}$ steps in a session. The subjects made four adjustments for each test stimulus. In the increasing series this procedure was repeated again with the reverse order of the spectrum. In total, 8 adjustments for brightness matching were performed for each wavelength under each retinal illuminance level, and the mean relative energy to make a brightness match was obtained. At the beginning of each session the subjects were dark adapted for 
10 to 30 minutes depending on the retinal illuminance level.

Seven subjects with normal color vision, who were checked by the Ishihara Test, participated in the experiment. The subjects ranged in age from 19 years to 23 years.

\section{Results}

Figure 3 shows the results of individual subjects in each of seven panels respectively. The abscissa indicates the wavelength of the test stimulus and the ordinate the luminous efficiency (in log unit). The luminous efficiency is given by the reciprocal of relative energy required to make a brightness match, so that the efficiency becomes higher as the retinal illuminance of the reference stimulus decreases. The top curve in each panel is for the $0.01 \mathrm{td}$ level and the bottom one for the $100 \mathrm{td}$ level. As the retinal illuminance increases, the efficiency function changes from the top to the bottom of the figure. It is noted that the luminous efficiency defined here, the reciprocal of the relative energy to make a brightness match, is expressed in an arbitrary unit for each efficiency curve. Then it is nothing meaningful that, for example, the efficiency for the $0.01 \mathrm{td}$ level is about $4 \mathrm{log}$ unit higher than that for the $100 \mathrm{td}$ level as expressed in the figure. It simply means that the $0.01 \mathrm{td}$ curve is shifted up along the ordinate by an amount of the brightness difference between the $100 \mathrm{td}$ and the $0.01 \mathrm{td}$ levels. Though the efficiency is expressed in arbitrary units, the relative difference within a set of nine curves in each subject is meaningful since the measurement was carried out with same apparatus and same calibration system. Then to normalize a set of nine curves for each subject, all the nine curves are equally shifted along the ordinate so as to equate the only one data point, the $510 \mathrm{~nm}$ point in the $0.01 \mathrm{td}$ level, to 6.0 .

As the retinal illuminance decreases, the efficiency curve gradually changes its shape in a manner that the efficiencies in short-wave region are relatively hightened in comparison with those in long-wave region. Thus a shift of the peak toward the shorter-wave region, that is so-called the Purkinje shift, is seen. Such property was also reported in previous studies ${ }^{1)} 3$. Although a different manner can be seen for HM, the extent of the change is almost same for all the subjects.

To see the individual variation for each retinal illuminance level the data points in Fig. 3 are replotted in Fig. 4 according to each retinal illuminance. The each data point indicates the individual result and the solid line is the average of seven subjects. The average is taken as a geometric mean, that is, the simple arithmetic mean of $\log$ efficiency data. The variations are rather large except for the lowest two levels. Particularly for the 3.2 to 0.32 td range fairly large variations
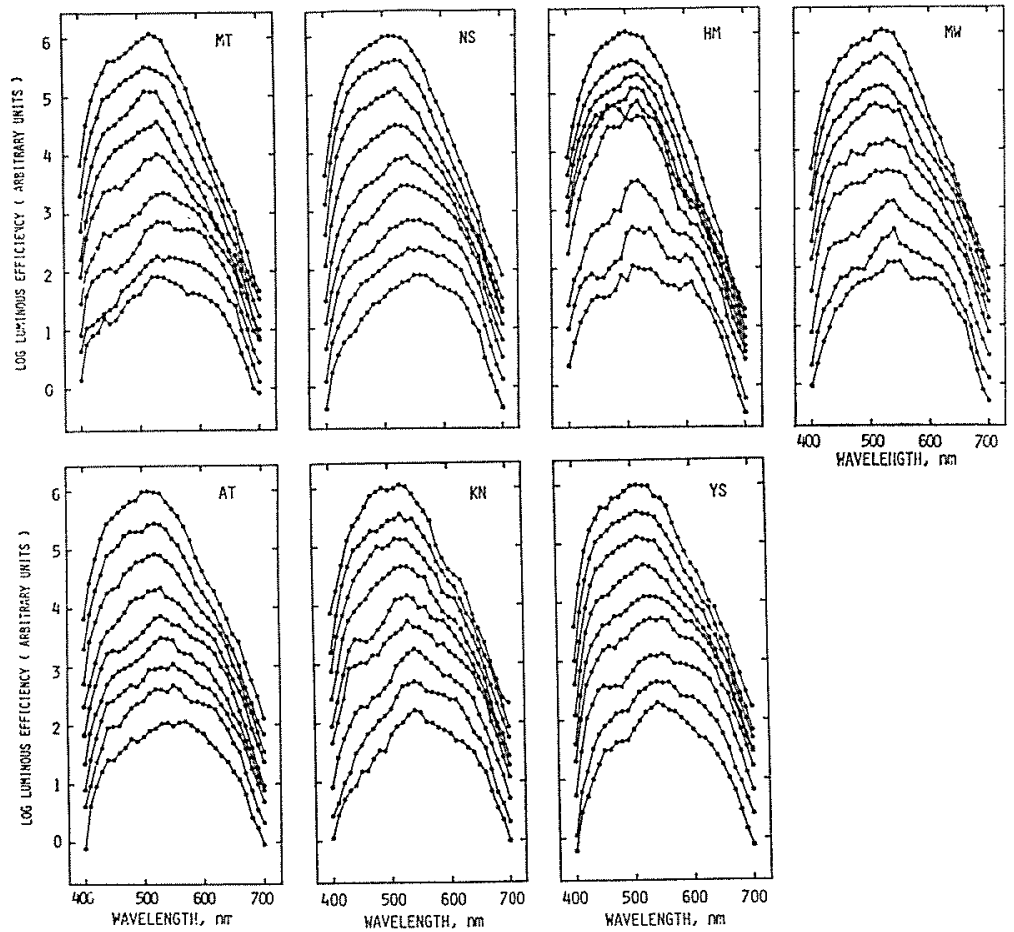

Fig. 3 Spectral luminous efficiency functions at nine retinal illuminance levels from 0.01 td to $100 \mathrm{td}$ in $0.5 \mathrm{log}$ unit steps for seven subjects. The top curve corresponds to the 0.01 td level and the bottom one to the $100 \mathrm{td}$ level. Retinal illuminance increases as going from the top to the bottom of the every figures. The $510 \mathrm{~nm}$ data at the 0.01 td level are normalized to 6.0. 


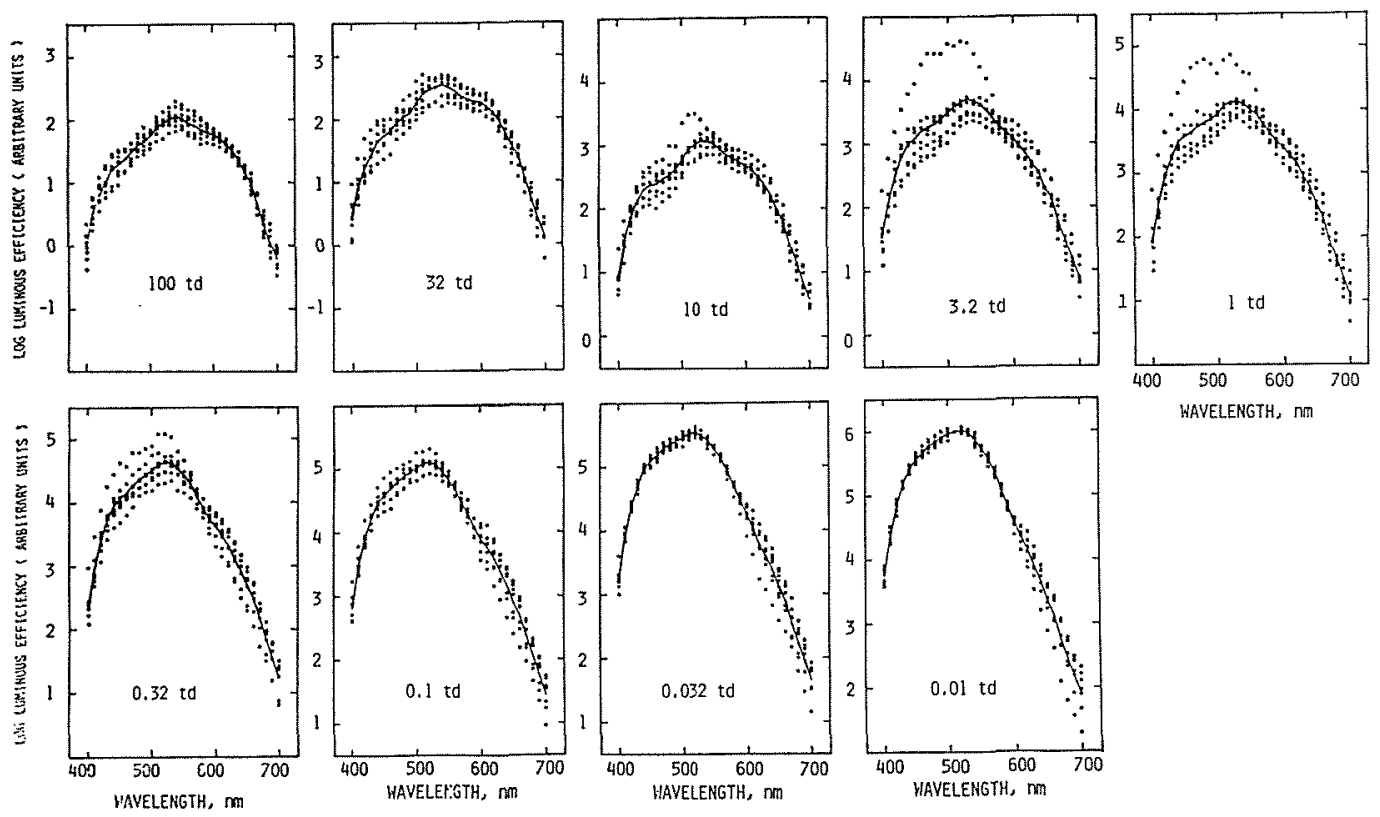

Fig. 4 Individual variation of luminous efficiency function for each retinal illuminance level. Closed symbols indicate the individual results replotted from Fig. 3 and the solid lines are the average functions of seven subjects taken by geometric mean.

can be seen, where the transition from the photopic function to the scotopic one remarkably occurs. For the lowest two levels, the 0.032 and $0.01 \mathrm{td}$ levels, the variations are fairly small except for the longwave region. It suggests that in these two levels the rod system is mainly responsible for brightness perception, while in the other higher levels the three types of cone systems and also rod system are active, and these systems make different contributions for different subjects.

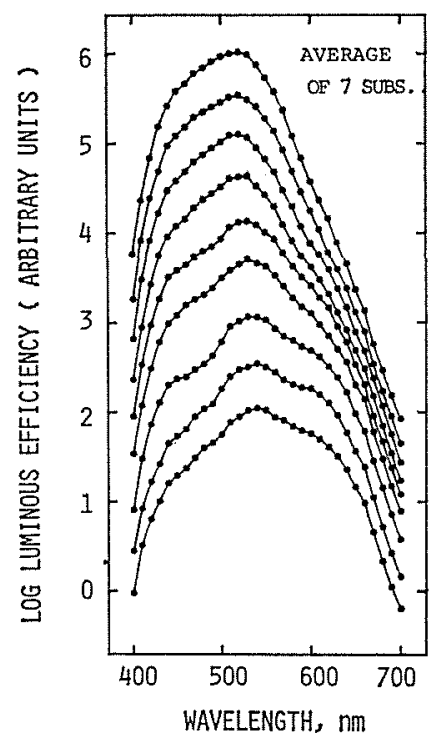

Fig. 5 Average luminous efficiency functions of 7 subjects at 9 retinal illuminance levels between 0.01 and $100 \mathrm{td}$.
Throughout the retinal illuminance levels investigated, it is shown that the individual variations in the mesopic range is fairly large compared to those in the photopic and scotopic ranges. However the change in the function with retinal illuminance systematically occurs as shown by the solid lines in Fig. 4. Then it is possible to establish the average functions for all the levels. Figure 5 shows these average functions. It is obvious that the function shows continuous change with retinal illuminance and all the curves differ in shape from each other. There is no representative efficiency function in the mesopic range. Though the number of subjects may be still short for the standardization, the present data could be served as a tentative standard data. The numerical values of the efficiency data are shown in Table 1 in $\log$ unit.

\section{Discussion}

(1) The luminous efficiency functions for $a 10^{\circ}$ field at photopic and scotopic vision.

In order to connect the mesopic photometry system for a large visual field such as $10^{\circ}$ field to the photopic and scotopic systems, it is important to determine the basic efficiency functions for a large field at photopic and scotopic vision, which have not been established yet. The CIE has recommended to use $\bar{y}_{10}(\lambda)$ function for a large field photometry at photopic, but it is based on the flicker photometry and color matching experiments and, therefore, is not appropriate to use for brightness perception. In the present results, the functions at the 100 and $0.01 \mathrm{td}$ levels are considered 
Table 1 Average huminous efficiency functions of seven subjects at nine retinal illuminance levels between 100 and $0.01 \mathrm{td}$ (in $\log$ anit). The $510 \mathrm{~nm}$ data at the 0.01 te level is normalized to 6.0 .

\begin{tabular}{|c|c|c|c|c|c|c|c|c|c|}
\hline \multirow{2}{*}{ Wavelength } & \multicolumn{9}{|c|}{ Retinal illuminance (td) } \\
\hline & 100 & 32 & 10 & 3.2 & 1 & 0.32 & 0.1 & 0.032 & 0.01 \\
\hline 400 & -.025 & .452 & .916 & 1.538 & 1.955 & 2.366 & 2.827 & 3.263 & 3.768 \\
\hline 410 & .517 & .927 & 1.483 & 2.080 & 2.533 & 2.950 & 3.488 & 3.915 & 4.368 \\
\hline 420 & .811 & 1.228 & 1.863 & 2.487 & 2.973 & 3.432 & 3.917 & 4.389 & 4.845 \\
\hline 430 & 1.010 & 1.428 & 2.113 & 2.783 & 3.267 & 3.752 & 4.225 & 4.695 & 5. 190 \\
\hline 440 & 1.207 & 1.654 & 2.305 & 2.990 & 3.510 & 3.959 & 4.477 & 4.983 & 5.422 \\
\hline 450 & 1.292 & 1.733 & 2.372 & 3.081 & 3.587 & 4.078 & 4.583 & 5.088 & 5.582 \\
\hline 460 & 1. 374 & 1.816 & 2.399 & 3.196 & 3.649 & 4.155 & 4.690 & 5.188 & 5. 666 \\
\hline 470 & 1.490 & 1.953 & 2.477 & 3.258 & 3.735 & 4.284 & 4.796 & 5.293 & 5.780 \\
\hline 480 & 1.605 & 2.036 & 2.541 & 3.309 & 3.797 & 4.377 & 4.875 & 5.353 & 5.842 \\
\hline 490 & 1.660 & 2.092 & 2.634 & 3.387 & 3.849 & 4.443 & 4.950 & 5.408 & 5.912 \\
\hline 500 & 1.749 & 2.259 & 2.788 & 3.500 & 3.936 & 4.518 & 5.018 & 5.458 & 5.970 \\
\hline 510 & 1.887 & 2.395 & 2.959 & 3.584 & 4.066 & 4.609 & 5.094 & 5.518 & 6.000 \\
\hline 520 & 1.949 & 2.477 & 3.016 & 3.654 & 4.120 & 4.629 & 5.106 & 5.537 & 6.018 \\
\hline 530 & 2.019 & 2.506 & 3.066 & 3.709 & 4.136 & 4.634 & 5.069 & 5. 491 & 5.991 \\
\hline 540 & 2.049 & 2. 547 & 3.057 & 3.665 & 4.095 & 4.528 & 4.960 & 5.416 & 5.877 \\
\hline 550 & 2.024 . & 2.503 & 3.032 & 3.629 & 4.010 & 4.433 & 4.829 & 5.283 & 5.736 \\
\hline 560 & 1.948 & 2.448 & 2.936 & 3.529 & 3.935 & 4.298 & 4.688 & 5.148 & 5.578 \\
\hline 570 & 1.909 & 2.362 & 2.848 & 3.409 & 3.779 & 4.097 & 4.483 & 4.936 & 5.372 \\
\hline 580 & 1.841 & 2.312 & 2.801 & 3.275 & 3.630 & 3.917 & 4.300 & 4.701 & 5.087 \\
\hline 590 & 1. 794 & 2.275 & 2.736 & 3.177 & 3.513 & 3.748 & 4.079 & 4.467 & 4.841 \\
\hline 600 & 1.761 & 2.263 & 2.689 & 3.105 & 3.415 & 3.627 & 3.889 & 4.255 & 4.579 \\
\hline 610 & 1.697 & 2.198 & 2.619 & 2.981 & 3.282 & 3.484 & 3.761 & 4.041 & 4.375 \\
\hline 620 & 1.616 & 2.105 & 2.513 & 2.853 & 3.167 & 3.320 & 3.596 & 3.787 & 4.171 \\
\hline 630 & 1. 510 & 1.967 & 2.392 & 2.708 & 2.959 & 3.115 & 3.385 & 3.605 & 3.897 \\
\hline 640 & 1.364 & 1.775 & 2.224 & 2.563 & 2.788 & 2.926 & 3.175 & 3.391 & 3. 663 \\
\hline 650 & 1.167 & 1.571 & 1.985 & 2.307 & 2.535 & 2.693 & 2.900 & 3.121 & 3. 372 \\
\hline 660 & .991 & 1.392 & 1.794 & 2.150 & 2.311 & 2.491 & 2.687 & 2.905 & 3. 140 \\
\hline 670 & .666 & 1.056 & 1.461 & 1.785 & 1.964 & 2.187 & 2.386 & 2.544 & 2.771 \\
\hline 680 & .339 & .721 & 1.162 & 1.480 & 1.678 & 1.854 & 2.078 & 2.242 & 2. 479 \\
\hline 690 & .052 & .430 & .868 & 1. 179 & 1.387 & 1.551 & 1.764 & 1.962 & 2.191 \\
\hline 700 & -.194 & .170 & .587 & .899 & 1.086 & 1.243 & 1.446 & 1.660 & 1. 933 \\
\hline
\end{tabular}

to be photopic and scotopic functions respectively. These two functions could be used as representative functions for a large field at photopic and scotopic visions.

For photopic vision, the brightness luminous efficiency function for a $2^{\circ}$ field was reported by the CIE TC-1.47). Recently Ikeda, Yaguchi, and Sagawa ${ }^{8)}$ reported more reliable one which modified the TC-1.4 report. To compare the present photopic function with the $2^{\circ}$ brightness function, the average data at the $100 \mathrm{td}$ level in Fig. 5 are replotted in Fig. 6 as open circles connected by solid lines together with the modified TC-1.4 data by Ikeda et al. as a dashed line. Both data are normalized at $570 \mathrm{~nm}$ as usual. The two curves almost agree with each other, and there is no doubt that the 100 td level examined here is photopic. For middleand long-wave regions the $2^{\circ}$ and $10^{\circ}$ functions are in good agreement though a slight difference at around $600 \mathrm{~nm}$ is seen. However, the significant difference is observed for the short-wave region where the $10^{\circ}$ function shows higher efficiency than the $2^{\circ}$. This difference can be considered due to the macular pigmentation. To test this, the efficiency differences between the $10^{\circ}$ and $2^{\circ}$ data are calculated, which are plotted as open circles in the inset of Fig. 6. The dashed line in the inset represents the spectral density of the macular pigment by Wyszecki and Stiles'). Good fit can be observed. This suggests that we can use the $2^{\circ}$ data as a large field function approximately with the correction of macular pigment density. However, some discrepancies between the $2^{\circ}$ and $10^{\circ}$ functions which cannot be simply explained by the macular pigmentation still remain, and this means the necessity of establishing a $10^{\circ}$ function by further collection of experimental data.

For scotopic vision, as we know, there exists already standardized CIE $V^{\prime}(\lambda)$ function. We compare the present results obtained at the lowest retinal illuminance level with the $V^{\prime}(\lambda)$ function in Fig. 7. The open circles connected by solid lines are the present 0.01 td data and the dashed line the $V^{\prime}(\lambda)$ function both normalized at $510 \mathrm{~nm}$. It is shown that the present 0.01 td function almost agrees with the $V^{\prime}(\lambda)$ function. However two noteworthy differences can be seen. One is the hightened efficiency of the $0.01 \mathrm{td}$ data in long-wave region where cone system is still operative since all the subjects reported red color sensation in this part of the spectrum. The other is a systematic difference from $V^{\prime}(\lambda)$ in short- and middle-wave regions: the pre- 


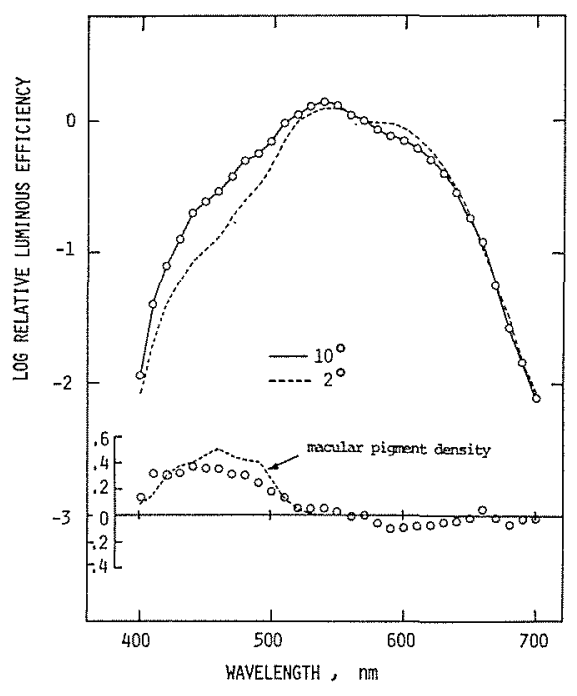

Fig. 6 Comparison of the $10^{\circ}$ and $2^{\circ}$ brightness functions for photopic vision. The $10^{\circ}$ function is the present 100 td data (open circles) and the $2^{\circ}$ function is from Ikeda et al. (dashed line). Inset: The log efficiency differences between the $10^{\circ}$ and $2^{\circ}$ data (open circles) and the macular pigment density curve from $W y s z e c k i$ and Stiles (dashed line).

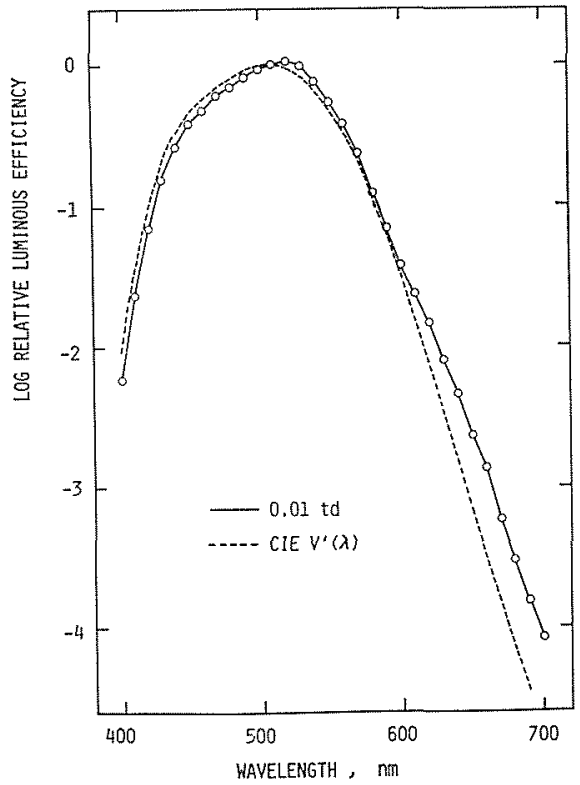

Fig. 7 Comparison of the present 0.01 td data (open circles connected by solid lines) with the CIE scotopic function $V^{\prime}(\lambda)$ (dashed line).

sent $0.01 \mathrm{td}$ curve slightly shifts to the long-wave direction so that it has a peak at $520 \mathrm{~nm}$. It is anxious that the lowest level we examined here is not fully in scotopic vision and the discrepancies from the $V^{\prime}(\lambda)$ might disappear if the retinal illuminance further decreases. However, precise comparison between the $0.032 \mathrm{td}$ and the $0.01 \mathrm{td}$ data reveals no significant difference except for the long-wave region where slight differences can be observed. Then the discrepancies from the $V^{\prime}(\lambda)$ mentioned above are essential for the centrally viewed $10^{\circ}$ field below the $0.032 \mathrm{td}$ level. This means that the CIE $V^{\prime}(\lambda)$ function can not necessarily be regarded as a standard data for a large field which is viewed centrally, and then we need another standard function, like the present $0.01 \mathrm{td}$ data, for a centrally viewed large visual field in scotopic vision.

\section{(2) The luminous efficiency functions at mesopic vision}

As seen in Fig. 5 the efficiency curves between the $100 \mathrm{td}$ and the $0.01 \mathrm{td}$ levels change their shapes gradually from the photopic to the scotopic function with the decrease of retinal illuminance level. As we mentioned before, there is no representative function in the mesopic range. Therefore, the mesopic luminous efficiency function should be established for all the retinal illuminance levels.

To evaluate the change in the function from the photopic function, the relative difference in efficiency curve in Fig. 5 between each retinal illuminance level and the 100 td level is calculated and the results are shown in Fig. 8. The top curve, for example, shows higher efficiency for short-wave stimulus by about $2 \log$ unit than that for long-wave stimulus compared to the photopic function. The same tendency is seen for other retinal illuminance levels, though less pronounced as the retinal illuminance increases. On the whole, the change in the function from the photopic is characterized by that the lower the retinal illuminance the more hightened the efficiency for shortwave region.

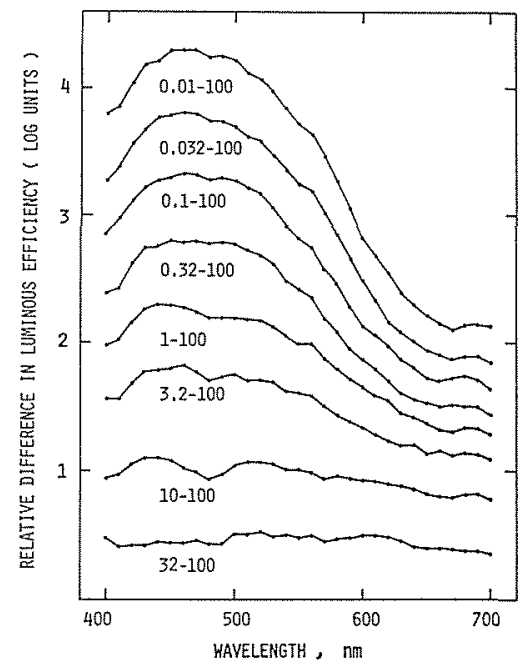

Fig. 8 Relative difference of luminous efficiency at each retinal illuminance level from the one at $100 \mathrm{td}$ level calculated from Fig. 5 (in log unit). 
It is interesting to know at what retinal illuminance level the mesopic change in efficiency function occurs. The bottom curve in Fig. 8 , the log difference between the 32 and 100 td data, shows almost flat along the spectrum, and this indicates that the function at the $32 \mathrm{td}$ is exactly same in shape as that at the $100 \mathrm{td}$. But, as shown in the next curve to the bottom in Fig. 8 , the change from the photopic function occurs at the 10 td level; the level which we usually considered as photopic. Therefore, it can be said that the mesopic vision starts at the 10 td level when decreasing the retinal illuminance. If we assume the pupil area is $10 \mathrm{~mm}^{2}$ this level corresponds to $1 \mathrm{~cd} / \mathrm{m}^{2}$. Below this level, the luminous efficiency function differs gradually from the photopic function until it finally reaches to the scotopic function. And below the $0.1 \mathrm{td}$ level the functions become basically the scotopic-type. The efficiency change in mesopic vision is pronounced particularly for the range between the 3.2 and $0.32 \mathrm{td}$ levels.

\section{(3) Contributions of photopic and scotopic functions to mesopic runctions}

It is important for the photometry to represent the efficiency change in mesopic vision quantitatively by a simple formula. It is generally considered that the change is due to the relative contribution of rod and cone systems. Some models to express the mesopic functions with photopic and scotopic functions have been reported ${ }^{4) \sim 6)}$. Among them the Ikeda and Shimozono's formula $\left.{ }^{6}\right)$ shows fairly good fits to the experimental data by using the photopic function determined by direct matching method instead of the function based on flicker photometry which the other models use. Their model is as follows:

$$
\log S_{T}(\lambda)=a \log S_{L}(\lambda)+b \log S_{C}(\lambda)+c \cdots \cdots \cdots(1)
$$

where $S_{r}(\lambda)$ is a theoretical mesopic function and $S_{k}(\lambda)$, and $S_{c}(\lambda)$ are the scotopic and photopic functions, respectively, determined experimentally: for example the top and the bottom curves in Fig. 3 or Fig. 5. The coefficients $a$ and $b$ can be regarded as the relative contribution of rod and cone systems respectively and $c$ is a constant to determine the efficiency level.

Fig. 9 shows the theoretical functions calculated by the Ikeda-Shimozono's formula with a least square method as solid lines together with the experimental data as closed symbols for subject $M W$ and HM, for example, and for the average data. Of course for the top and the bottom curves in all the panels, the theoretical and the experimental data are exactly the same as expected from equation (1). For intermediate levels, the theoretical data show good fits to the experimental ones. Particularly, fits are excellent for the average data. The coefficients $a$ and $b$ for the average data are shown in Fig. 10 as a function of log retinal illuminance, and the numerical values $a, b$, and $c$ are also shown in Table 2. The coefficient a, which means the the rod system contribution, decreases as the retinal

Table 2 The coefficients $a, b$ and $c$ in Ikeda-Shimzono's formula to give best fits to the average data of seven subjects

\begin{tabular}{c|c|c|c|c|c|c|c|c|c}
\hline \multirow{2}{*}{ Coefficient } & \multicolumn{7}{|c}{ Retinal illuminance (td) } \\
\cline { 2 - 8 } & 100 & 32 & 10 & 3.2 & 1 & 0.32 & 0.1 & 0.032 & 0.01 \\
\hline $\mathrm{a}$ & 0 & .012 & .099 & .296 & .427 & .610 & .756 & .888 & 1 \\
$\mathrm{~b}$ & 1 & 1.037 & .925 & .715 & .586 & .390 & .226 & .104 & 0 \\
$\mathrm{c}$ & 0 & .359 & .609 & .495 & .418 & .168 & .080 & -.011 & 0 \\
\hline
\end{tabular}
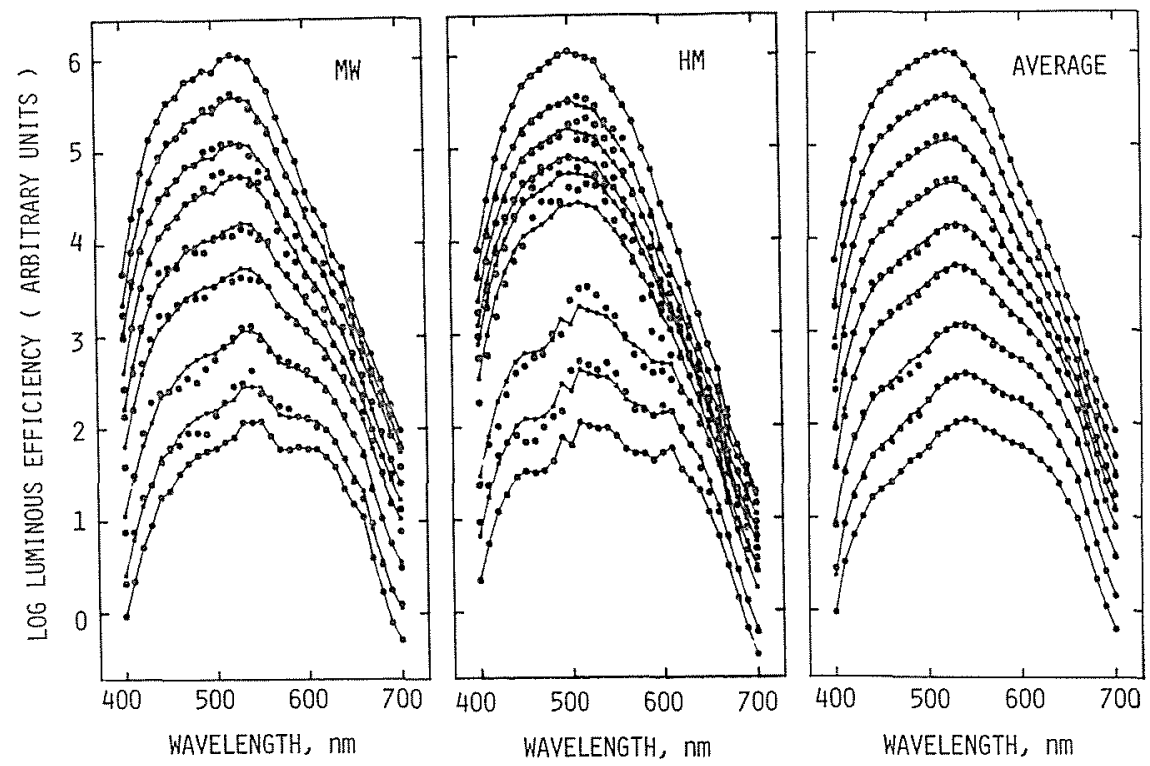

Fig. 9 Comparison of the present data (closed circles) with the theoretical efficiency functions derived by the Ikeda-Shimozono's formula (solid lines) for subjects $M W$ and $H M$, and for the average data. 


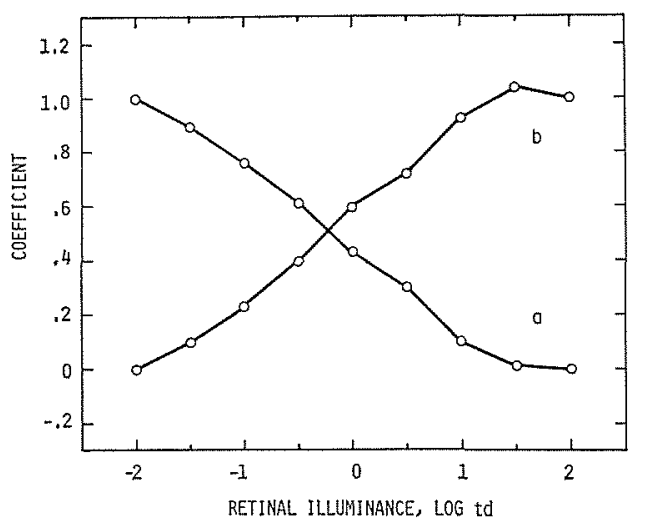

Fig. 10 The coefficients $a$ and $b$ in the Ikeda-Shimozono's formula to give best fits to the present average functions. The coefficients are represented as a function of log retinal illuminance.

illuminance level increased, on the contrary $b$ increases with retinal illuminance. The value $a+b$ is close to unity for all the levels. It means that the contributions of rod and cone systems are complementary. As shown in Fig. 10, the coefficient $b$ shows almost plateau at 1.0 above $1.5 \log$ td ( $32 \mathrm{td}$, which means only the cone system contributes to the brightness perception for the levels higher than the $32 \mathrm{td}$. The notion mentioned above that the efficiency change in mesopic vision occurs at first at the 10 td level is in good agreement with the results of this analysis.

\section{Conclusions}

The present study have shown an extensive experimental data for mesopic luminous efficiency functions based on direct brightness matching of $10^{\circ}$ field for a wide luminance range covering photopic, mesopic, and scotopic vision. As shown in Fig. 5, the efficiency functions change continu- ously from the photopic function to the scotopic with decreasing of retinal illuminance. This shows that we should establish the standard mesopic functions for all the luminance levels. The present data can provide the basis of such a standard. The collection of data will be continued further in order to establish a standard of mesopic luminous efficiency functions.

\section{Acknowledgments}

We thank Prof. Mitsuo Ikeda at Tokyo Institute of Technology for his valuable comments on this research. Thanks are also to $\mathrm{Mr}$. Tadashi Masuda at Industrial Products Research Institute for his help with computer programming in data analysis. This research was supported by a grant of Ministry of International Trade and Industry.

\section{References}

(1) Kinney, J. A. S.: "Comparison of Scotopic, Mesopic, and Photopic Spectral Sensitivity Curves." J. Opt. Soc. Am. 48 (1958) 185 190.

(2) Walters, H. V. and Wright, W. D.: "The spectral sensitivity of the fovea and extrafovea in the Purkinje range." Proc. Roy. Soc. Lond. B 131 (1943) 340 361.

(3) Kolxoschka, S.: "Untersuchungen zur mesopischen Strahlungsbewertung." Die Farbe. 21 (1972) 39 112.

(4) Palmer, D. A.: "The definition of a standard observer for mesopic photometry." Vision Res. 7 (1967) $619 \sim 628$

(5) Kokoschka, S. and Bodmann, H. W.: "Ein konsistentes System zur photometrischen Strahlungsbewertung im gesamten Adaptationsbereich." CIE Pub. No. 36 (1976) 217 225.

(6) Ikeda, M. and Shimozono, H.: "Mesopic Iuminousefficiency functions." J. Opt. Soc. Am. "y1 (1981) $280 \sim 284$.

(7) CIE TC-1.4: "Light as a true visual quantity: Principles of measurement." CIE Pub. No. 41 (1978) 36 38.

(8) Ikeda, M., Yaguchi, H. and Sagawa, K.: "Brightness luminous-efficiency functions for $2^{\circ}$ and $10^{\circ}$ fields." J. Opt. Soc. Am. 72 (1982) 1660 1665.

(9) Wyszecki, G. and Stiles, W. S.: Color Science (2nd ed.), 111 113 Wiley, New York (1982).

Received 7 Mar. 1983; Revision Received 11 Aug. 1983 